\title{
Laparoscopic end-to-end biliary reconstruction with T-tube for transected bile duct injury during laparoscopic cholecystectomy
}

\author{
Bong Jun Kwak, Ho Joong Choi, Young Kyoung You, Dong Goo Kim, Tae Ho Hong \\ Department of Hepatobiliary and Pancreas Surgery, Seoul St. Mary's Hospital, College of Medicine, The Catholic University of \\ Korea, Seoul, Korea
}

\begin{abstract}
Purpose: This report describes the laparoscopic end-to-end biliary reconstruction with T-tube for transected bile duct injury (BDI) during laparoscopic cholecystectomy.

Methods: We performed a retrospective descriptive analysis for all patients with a transected BDI at a single institution. We collected and analyzed data for injury site and type, reconstruction methods, conversion rate, previous intervention, and outcomes.

Results: Between January 2014 and December 2017, 2,901 patients underwent laparoscopic cholecystectomy at a single institution. Among them, 8 patients experienced a transected BDI during laparoscopic cholecystectomy, so the surgeon performed laparoscopic end-to-end biliary reconstruction with T-tube. Our patient series consisted of 6 women (75\%) and 2 men (25\%) with a mean age of 48.3 years (median, 49 years; range, 29-77 years). Two cases were converted to open surgery. The most common injured site was the common bile duct $(5$ of $8,62.5 \%)$. The most common injury type, using Bismuth's classification system, was type I ( 3 of $8,37.5 \%$ ). The mean operating time was 136.8 minutes (median, 135.0 minutes; range, 0-180.0 minutes). The mean hospital stay was 7.0 days (median, 4.5 days, range: $3.0-21.0$ days). The mean follow-up was 36.4 months (median, 34.0 months; range, 16.0-63.0 months). We observed one postoperative complication during the follow-up period. The patient had an anastomosis site leakage and was cured after reoperation.

Conclusion: Laparoscopic end-to-end biliary reconstruction with T-tube for transected BDI during laparoscopic cholecystectomy seems to be safe and feasible in selected patients. However, long-term follow-up to identify complications from bile duct stricture remains important.
\end{abstract}

[Ann Surg Treat Res 2019;96(6):319-325]

Key Words: Bile duct injury, Laparoscopy, Cholecystectomy, T-tube

\section{INTRODUCTION}

The first laparoscopic cholecystectomy was performed in 1985 and has become increasingly popular since the 1990s. Laparoscopic cholecystectomy is now the gold standard for benign gallbladder diseases, including cholelithiasis. Never- theless, in the 1990s many reports revealed a significant increase the incidence of bile duct injuries (BDIs) during laparoscopic cholecystectomy $(0.33 \%-1.09 \%)$ compared with open cholecystectomy $(0.20 \%-0.51 \%)$ [1]. In response, many effective methods for repairing and preventing BDIs have been introduced, such as intraoperative cholangiography and
Received July 27, 2018, Revised February 10, 2019, Accepted March 17, 2019

\section{Corresponding Author: Tae Ho Hong}

Department of Hepatobiliary and Pancreas Surgery, Seoul St. Mary's Hospital, College of Medicine, The Catholic University of Korea, 222 Banpo-daero, Seocho-gu, Seoul 06591, Korea

Tel: +82-2-2258-2876, Fax: +82-2-595-2822

E-mail: gshth@catholic.ac.kr

ORCID code: https://orcid.org/0000-0003-3864-8104
Copyright (c) 2019, the Korean Surgical Society

(c) Annals of Surgical Treatment and Research is an Open Access Journal. All articles are distributed under the terms of the Creative Commons Attribution NonCommercial License (http://creativecommons.org/licenses/by-nc/4.0/) which permits unrestricted non-commercial use, distribution, and reproduction in any medium, provided the original work is properly cited. 
allowing a better surgical view to reduce iatrogenic BDIs [2]. Unfortunately, recent studies reported that rates of BDI during laparoscopic cholecystectomy had plateaued $(0.30 \%-0.60 \%)$ [3]. One study suggested that $34 \%$ of BDIs during laparoscopic cholecystectomy occurred during surgeries performed by surgeons that had performed over 100 laparoscopic cholecystectomies [4], indicating that even expert surgeons can cause a major BDI requiring surgery.

BDI is associated with increased risk for several perioperative morbidities, including frequent bile duct stricture, recurrent cholangitis, decreased quality of life, and even death. Various injuries can occur along the biliary tract during laparoscopic cholecystectomy, and transected bile duct injury is one of the most commonly encountered. Importantly, it is not easy to reconstruct this type of injury. Currently, the Roux-en-Y choledocho (or hepatico)-jejunostomy is the most commonly used reconstruction procedure for transected bile duct injuries. However end-to-end reconstruction, which is a much simpler procedure can be performed safely and effectively in select patients [5,6]. In particular, due to the development of advanced laparoscopic techniques, laparoscopic reconstruction can be performed for BDI during surgery. Nevertheless, evaluations of laparoscopic end-to-end biliary reconstruction for transected $\mathrm{BDI}$ are very rare.

This report describes eight successful cases of laparoscopic end-to-end biliary reconstruction with T-tube that were performed for transected BDIs during laparoscopic cholecystectomy.

\section{METHODS}

\section{Patients}

Between January 2014 and December 2017, 2,901 patients underwent laparoscopic cholecystectomy for the benign biliary disease at the Hospital. The benign biliary diseases included polypoid gallbladder lesions, cholelithiasis, cholecystitis, choledocholithiasis, biliary dyskinesia, and acute pancreatitis.

We conducted a retrospective descriptive analysis of all patients who underwent surgery for transected BDIs $(n=8)$ at a single institution between January 2014 and December 2017. For each patient, we collected and analyzed demographic data, injury site and type, reconstruction methods, conversion to open surgery (yes or no), history of previous intervention, and surgical outcomes including operation time, hospital stay, and complications. We used the Bismuth classification system for classifying BDI type [2].

Our hospital is a tertiary education and training hospital with eight hepatobiliary surgeons on staff. Surgeons who performed laparoscopic cholecystectomy ranged from resident to professor; among them, Dr. Hong, who is a hepatobiliary surgeon, performed all the reconstruction surgeries related to BDI during laparoscopic cholecystectomy. The study was reviewed and approved by the Ethics Committee of the Seoul St. Mary's Hospital (KC18RCSI0341), which waived the requirement for informed consent due to the retrospective study design.

\section{Surgical technique}

Laparoscopic end-to-end anastomosis was performed when it was possible to approximate both the proximal and distal ends of bile duct without tension, and when the diameters of both ends were comparable. Both ductal ends must be healthy without visual evidence of inflammation, fibrosis, or ischemic damage according to the unaided eye. The Kocher maneuver to reduce tension at both ductal ends was not performed in any of these cases.

Step 1. After mobilization of both the proximal and distal bile ducts, damaged or narrowed bile duct ends were cleared. We placed 2 anchoring sutures at both corners of the bile ducts for approximation.

Step 2. Anastomosis was performed in a single layer with interrupted absorbable Monosyn 5-0 sutures at the posterior wall. Two or 3 stitches were needed for posterior wall anastomosis according to bile duct diameter.

Step 3. T-tube made of $8-10 \mathrm{~F}$ rubber was inserted at the anastomosis site.

Step 4. Two or 3 stitches were placed for completing anterior wall anastomosis around the T-tube. After a leakage test of the T-tube using saline or gentian violet dye, additional stitches over the T-tube were performed if needed (Fig. 1). The T-tube was drained externally, and a closed suction drain was placed in the subhepatic space to detect postoperative bleeding or bile leakage. The drain tube was removed 24-48 hours after surgery, depending on the clinical course.

\section{Follow-up}

All patients went home once the closed suction drain was removed, we removed the drain only after confirming there was no evidence of bile leakage. Among all 8 patients, 5 patients were discharged with bile bag draining through the T-tube. Two patients were discharged with a clamped T-tube without external drainage, because the amount of bile juice drained for a day is small, and the patients had no symptom after clamping. And one patient died due to the progression of acute myeloid leukemia at postoperative day 21 .

After discharge, cholangiography via T-tube was performed 2 times (at 4 weeks and 8 weeks after surgery). In 5 patients discharged with bile bag draining through the T-tube, if cholangiography showed no bile duct obstruction or leakage at four weeks after surgery, the T-tube was clamped. In 2 patients discharged with T-tube clamped, we also performed cholangiography at 4 weeks after surgery to evaluate whether there was bile duct obstruction or leakage. If cholangiography 
showed no bile duct obstruction or leakage, the T-tube remained clamped until 8 weeks after surgery. After checking the cholangiography at 8 weeks after surgery, T-tube was removed in all patients.

After the 8-week follow-up cholangiography, we removed the tube in our outpatient clinic (Fig. 2A, B). Biochemical data with or without imaging were checked at 4-month intervals during the first postoperative year with annual follow-up thereafter. Follow-up data were obtained from outpatient records. Symptoms, biochemical data, imaging results, longterm complications, postoperative diagnostics, and therapeutic interventions were reviewed.
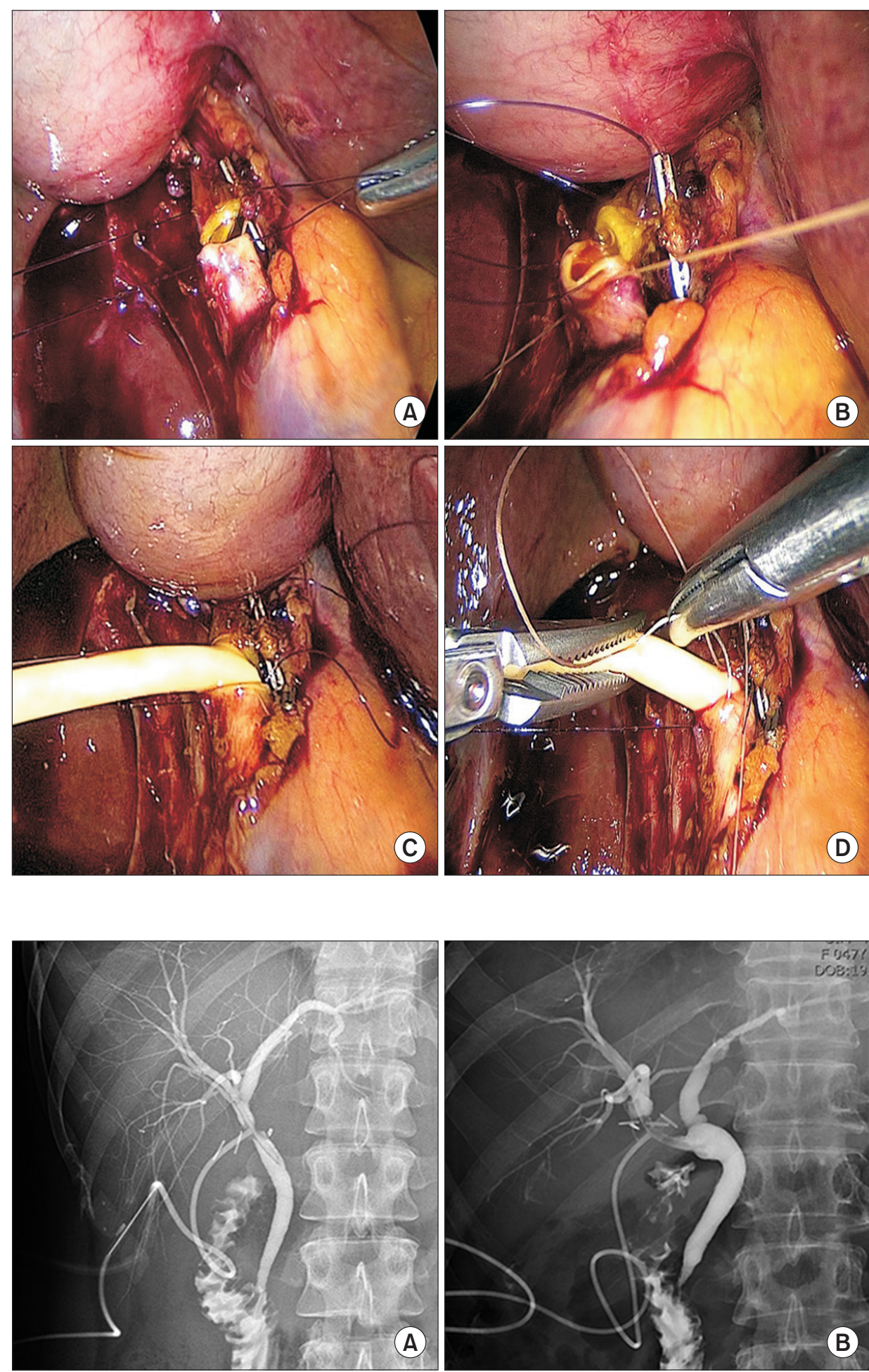

Fig. 2. Tubography at 4 weeks after end-to-end reconstruction with T-tube cholecystectomy; (A) reconstruction of common bile duct with T-tube; (B) reconstruction of the right hepatic duct with T-tube. 


\section{RESULTS}

During this period, 13 of the patients $(0.45 \%)$ were treated for iatrogenic BDIs that occurred during laparoscopic cholecystectomy. All 13 patients required repair or reconstruction of the bile duct and a single expert hepatobiliary surgeon performed all these procedures. Five patients had partial BDIs, and they received a laparoscopic primary repair. The other 8 patients had transected BDIs (Fig. 3), and they underwent laparoscopic end-to-end biliary reconstruction with T-tube insertion. Among these 8 cases, one was a 30-year-old female patient who was referred to our hospital after experiencing a transected BDI during laparoscopic cholecystectomy, for which she underwent laparoscopic end-to-end anastomosis of the right posterior hepatic duct (RPHD) with T-tube within 48 hours after the initial surgery. Another 12 patients were injured in our hospital during laparoscopic cholecystectomy, but all of these injuries were detected intraoperatively and were immediately treated by 1-stage surgery.

Our series consisted of 6 women (75\%) and 2 men (25\%) with a mean age of 48.3 years (median, 49 years; range, 29-77 years). We attempted laparoscopic end-to-end biliary reconstruction with T-tube insertion on all 8 patients that had an iatrogenic BDI during laparoscopic cholecystectomy. However, 2 cases were converted to open surgery. One case is a 30-year-old female patient who was referred to our hospital after transected BDI. In that case, we tried the laparoscopic approach but soon converted to open surgery by severe diffuse inflammation in the operation field due to bile leakage for more than 24 hours after initial laparoscopic cholecystectomy. Another case is a 51-year-old male patient who underwent percutaneous transhepatic gallbladder drainage (PTGBD) preoperatively. In

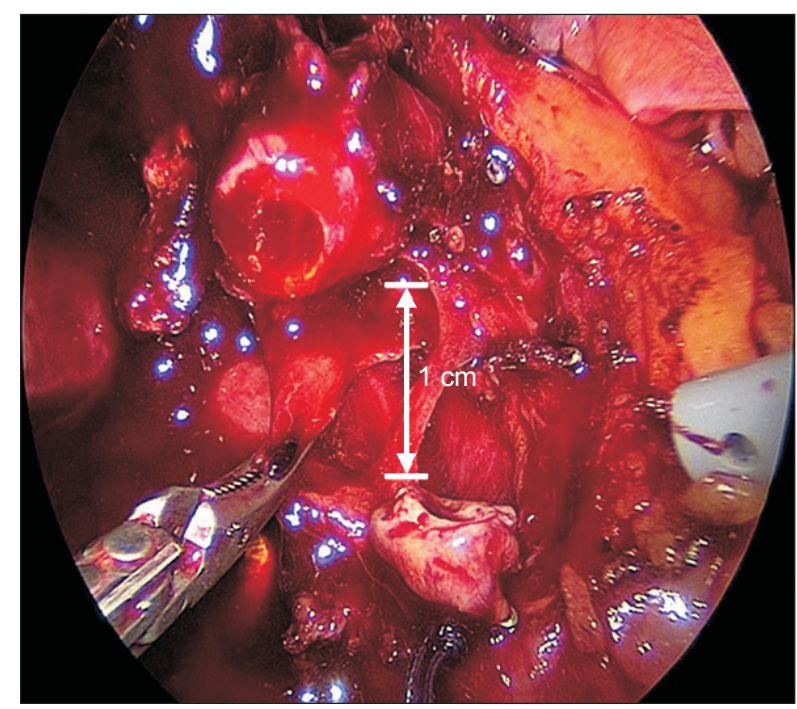

Fig. 3. Laparoscopic view of a transected common bile duct during laparoscopic cholecystectomy.

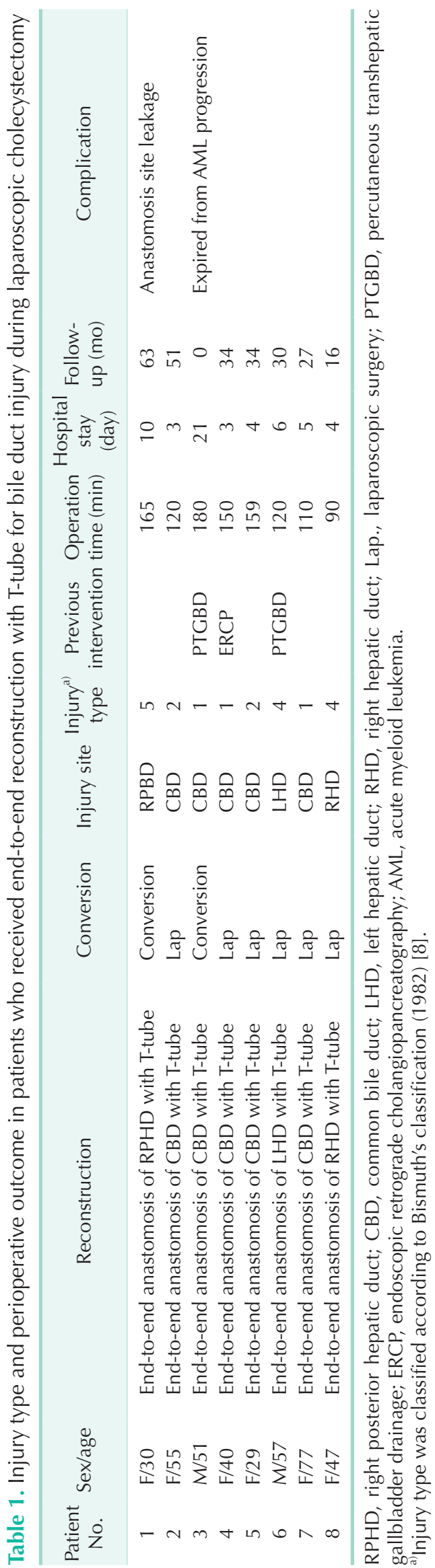


that case, we converted to open surgery by the difficulty of bleeding control at gallbladder bed. The most frequently injured site was the common bile duct (CBD) (5 of 8, 62.5\%). The other injury sites were the right hepatic duct (RHD; $n=1)$, the left hepatic duct (LHD; $\mathrm{n}=1$ ), and the RPHD $(\mathrm{n}=1)$ ). The most common injury type, according to Bismuth's classification, was type I ( 3 of $8,37.5 \%)$, followed by type II $(n=2)$, type IV ( $n=$ $2)$, and type $\mathrm{V}(\mathrm{n}=1)$, in that order. Three patients received preoperative interventions: 2 patients underwent PTGBD for severe gallbladder distension, and another underwent endoscopic retrograde cholangiopancreatography (ERCP) for a CBD stone.

The mean operating time was 136.8 minutes (median, 135 minutes; range, $0-180$ minutes). The mean hospital stay was 7.0 days (median, 4.5 days; range, 3.0-21.0 days). The mean duration of follow-up was 36.4 months (median, 34.0 months; range, 16.0-63.0 months). We observed one postoperative complication during the hospital stay. The patient had an anastomosis site leakage and was cured with the placement of an additional suture around the T-tube during reoperation on postoperative day 1 . We also observed one in-hospital mortality on a postoperative day 21. However, because the death was due to the progression of acute myeloid leukemia, and not surgical complications, we excluded this patient from our complications list (Table 1). Follow-up cholangiography or magnetic resonance image showed focal bile duct stricture without bile duct dilatation or obstruction at anastomosis site (Fig. 4). After discharge and all during the follow-up period, none of the 7 patients experienced any complications, including no evidence of bile leakage and no abnormal laboratory findings. Furthermore, even after T-tube removal later in the follow-

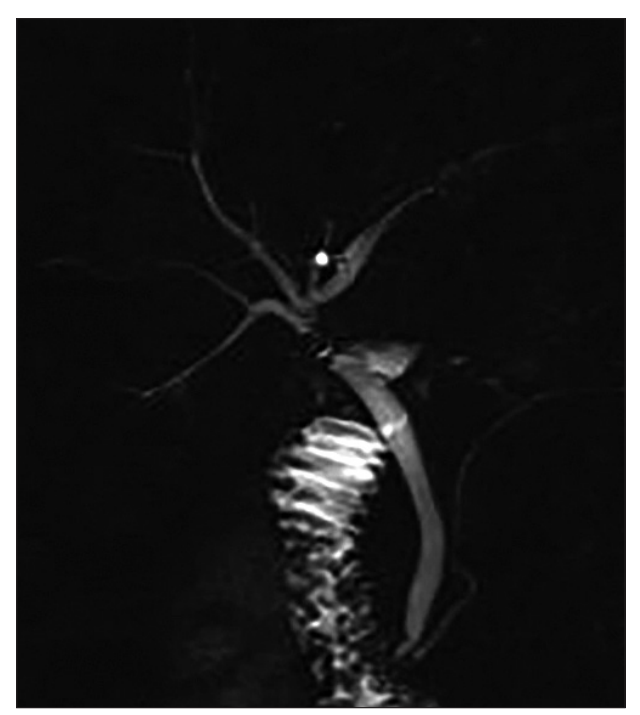

Fig. 4. Follow-up image at postoperative 5 months after T-tube removal. It showed focal bile duct stricture at anastomosis site without bile duct dilatation or obstruction. up period, no abnormalities requiring surgical or endoscopic treatment were observed.

\section{DISCUSSION}

There are many types of BDI for which various classifications have been proposed [7]. Bismuth's classification (1982) [8] and Strasberg's classification (1995) are the most commonly used systems [2], and, herein, we classified transected BDIs according to Bismuth's classification. The most frequently injured site among our study cases was CBD, with other injury sites being RHD, LHD, and RPHD. We classified the RHD injury and the LHD injury as type-IV injuries. The patients had anatomical variations in which the RHD and the LHD joined far from the hilum (Fig. 2B). In general, a focal injury of the bile duct during laparoscopic cholecystectomy can be treated with a laparoscopic primary closure without T-tube insertion. Otherwise, a transected BDI requires reconstructive surgery. Different biliary reconstructions for surgical treatment of iatrogenic BDI have been discussed: Rouxen-Y hepaticojejunostomy, end-to-end biliary anastomosis, choledochoduodenostomy, Lahey hepaticojejunostomy, jejunal interposition hepaticoduodenostomy, Blumgart (Hepp) anastomosis, Heinecke-Mikulicz biliary plastic reconstruction, and Smith mucosal graft.

Traditionally, most of transected BDIs have been treated by Roux-en-Y choledochojejunostomy (or hepaticojejunostomy) because end-to-end biliary anastomosis of a transected BDI is generally thought to increase the risk for anastomosis stricture and, consequently, high incidence of secondary repair [9]. Otherwise, currently, end-to-end anastomosis of the bile duct is the standard procedure for biliary reconstruction, including both living donor liver transplantation and deceased donor liver transplantation in adults. Some studies also suggested that endto-end biliary reconstruction after BDI yielded similar shortterm and long-term outcomes [5]. Consistent with previous findings, we performed eight cases of laparoscopic end-to-end biliary reconstruction for transected BDI during laparoscopic cholecystectomy, and we observed favorable short-term and long-term outcomes for our patients.

End-to-end biliary reconstruction has some definite advantages over many other bilioenteric anastomosis methods. First, it is a relatively easy and straightforward reconstruction method and is more physiological than Roux-en-Y choledochojejunostomy. After end-to-end biliary reconstruction, physiological bile flow is maintained through the intact ampulla of Vater, and ERCP can be performed after surgery if needed. Jablonska et al. [5] reported that significantly higher weight gain, lower early postoperative complications, and similar stricture rates were observed in patients undergoing end-to-end anastomosis compared with Roux-en-Y 
hepaticojejunostomy. The preferred lower weight gain in the end-to-end anastomosis group may be associated with better maintenance of the physiological course of the gastrointestinal tract and consequent proper absorption. Additionally, unlike with the Roux-en-Y choledochojejunostomy, there is no danger of intestinal obstruction by transmesocolic herniation or jejunal stump problems with end-to-end biliary reconstruction.

The biggest and most important previously described obstacle for end-to-end biliary reconstruction is bile duct stricture in long-term follow-up [9]. Even though the follow-up image showed focal stricture at the anastomosis site, we did not observe any bile duct dilatation or obstruction after the laparoscopic end-to-end biliary reconstructions with a T-tube drain. Jablonska et al. [5] reported similar anastomosis stricture rates $(9.68 \%$ vs. $5.26 \%, P=0.651)$ for end-to-end anastomosis and hepaticojejunostomy anastomosis after open and laparoscopic cholecystectomy, choledochotomy, and partial gastric resection. Kohneh Shahri et al. [6] also reported comparable success rates between end-to-end anastomosis and Roux-en-Y biliary reconstruction after laparoscopic cholecystectomy, although the number of participants was small. In another study of patients who were referred for treatment for complications after primary end-to-end biliary reconstruction for BDI, $66 \%$ were adequately managed by endoscopic or percutaneous stent placement, and 32\% underwent hepaticojejunostomy; the morbidity rate was low (5\%), and there were no mortalities. The researchers concluded that end-to-end anastomosis could be an initial treatment option when transected BDI without extensive tissue loss is detected perioperatively [10].

T-tube is one of the most commonly used materials to secure biliary anastomosis in BDI cases [5]. Otherwise, T-tube use for end-to-end biliary reconstruction is controversial. In this study, we maintained the placement of a rubbery T-tube for at least eight weeks. After removal, we observed focal bile duct stricture at the anastomosis site. Otherwise, there was no bile duct dilatation or obstruction in any cases. We suspect that the T-tube allows the bile duct diameter to be maintained while tissue fibrosis proceeds at the anastomosis site during the healing process. In this way, the T-tube acts as casting in the conduit of the bile duct. Furthermore, even a tiny amount of bile leakage at the anastomosis site can trigger bile duct stricture by aggravating periductal inflammation. External diversion of bile juice through the T-tube can eliminate the risk of any bile leakage at the anastomosis site. Based on these rationales, we have used T-tube in all end-to-end bile ducts anastomosis.

End-to-end biliary reconstruction is not appropriate for all transected BDI cases during laparoscopic cholecystectomy. The first condition required for successful implementation of the end-to-end anastomosis is that the anastomosed edges should be healthy without ischemia, inflammation, or fibrosis. In latedetected BDI, like cases identified 3 to 4 days after surgery, end-to-end biliary reconstruction may be impossible because sustained bile leakage may have made the proximal and distal bile ducts friable due to severe inflammation and bile contamination. Thus, early recognition and reconstruction of BDI, as in our cases, is essential for a good prognosis. Second, a tension-free anastomosis is important because excessive tension at an anastomosis site is a definite risk factor for anastomosis failure. Additionally, segmental loss of the bile duct conduit sometimes makes sufficient approximation of both the proximal and distal bile-duct ends impossible. If a tensionfree anastomosis cannot be performed, the only other option is to perform Roux-en-Y choledochojejunostomy for biliary reconstruction. Third, there should be no combined major vascular injuries. If a transected BDI co-occurs with a major vascular injury, hepatectomy, or even liver transplantation, may be necessary. In such cases, no reconstruction methods for bilioenteric anastomosis, including end-to-end biliary reconstruction, can be performed safely. Furthermore, it is vital to preserve, as much as possible, the axial arteries within the bile duct wall during dissection for reconstruction.

If transected BDI is detected intraoperatively during laparoscopic cholecystectomy and if it meets the conditions mentioned above, end-to-end biliary reconstruction with T-tube should be considered. A laparoscopic approach may also be possible if the surgeon has expertise in hepatobiliary and laparoscopic surgery.

In conclusion, laparoscopic end-to-end biliary reconstruction with T-tube for transected BDI during laparoscopic cholecystectomy seems to be safe and feasible in selected patients. However, long-term follow-up to identify complications from bile duct stricture remains important.

\section{CONFLICTS OF INTEREST}

No potential conflict of interest relevant to this article was reported.

\section{REFERENCES}

1. Adamsen S, Hansen OH, Funch-Jensen P,

Schulze S, Stage JG, Wara P. Bile duct injury during laparoscopic cholecystectomy: a 
prospective nationwide series. J Am Coll Surg 1997;184:571-8.

2. Strasberg SM, Hertl M, Soper NJ. An analysis of the problem of biliary injury during laparoscopic cholecystectomy. J Am Coll Surg 1995;180:101-25.

3. Hamad MA, Nada AA, Abdel-Atty MY, Kawashti AS. Major biliary complications in 2,714 cases of laparoscopic cholecystectomy without intraoperative cholangiography: a multicenter retrospective study. Surg Endosc 2011;25:3747-51.

4. Gigot J, Etienne J, Aerts R, Wibin E, Dallemagne B, Deweer F, et al. The dramatic reality of biliary tract injury during laparoscopic cholecystectomy. An anonymous multicenter Belgian survey of 65 patients. Surg Endosc 1997;11:1171-8.

5. Jablonska B, Lampe P, Olakowski M, Gorka Z, Lekstan A, Gruszka T. Hepaticojejunostomy vs. end-to-end biliary reconstructions in the treatment of iatrogenic bile duct injuries. J Gastrointest Surg 2009;13:1084-93.

6. Kohneh Shahri N, Lasnier C, Paineau J. Bile duct injuries at laparoscopic cholecystectomy: early repair results. Ann Chir 2005;130:218-23.

7. Lau WY, Lai EC. Classification of iatrogenic bile duct injury. Hepatobiliary Pancreat
Dis Int 2007;6:459-63.

8. Bismuth H. Postoperative strictures of the bile duct. In: Blumgart LH. The biliary tracted. Edinburgh (Scotland): Churchill Livingstone; 1982. p. 209-18.

9. Stewart L, Way LW. Bile duct injuries during laparoscopic cholecystectomy. Factors that influence the results of treatment. Arch Surg 1995;130:1123-8.

10. de Reuver PR, Busch OR, Rauws EA, Lameris JS, van Gulik TM, Gouma DJ. Long-term results of a primary end-to-end anastomosis in peroperative detected bile duct injury. J Gastrointest Surg 2007;11: 296-302. 